\title{
Fluorescence in situ hybridisation detection of erbB2 amplification in breast cancer fine needle aspirates
}

\author{
D T McManus, A H Patterson, P Maxwell, $M$ W Humphreys, N H Anderson
}

\begin{abstract}
Aim-To develop a method for the detection of amplification of the erbB2 oncogene in breast cancer fine needle aspirates using fluorescence in situ hybridisation (FISH) and to compare amplification with immunohistochemical detection of the erbB2 protein.

Methods-A digoxigenin labelled probe to the erbB2 gene was hybridised to 15 aspirates prepared from operative breast cancer specimens. A chromosome 17 centromere probe was also hybridised to the aspirates either separately or in combination with the erbB2 probe. The aspirates were scored for erbB2 amplification and chromosome 17 centromere number. Subsequently, paraffin wax embedded sections of the tumours were stained with the antibody CB11 and scored for the presence of membrane staining.

Results-Three of the 15 tumour aspirates showed high level amplification of erbB2 detected by FISH. These three tumours also showed chromosome 17 polysomy and diffuse membrane staining by immunohistochemistry.

Conclusions-FISH can be used to detect erbB2 amplification in fine needle aspirates and results correlate with conventional immunohistochemical staining. Difficulties were encountered in the visualisation of the signals in non-amplified cases without the use of specialised digital imaging.
\end{abstract}

(F Clin Pathol: Mol Pathol 1999;52:75-77)

Immunohistochemistry and Molecular Pathology Laboratory, Department of Pathology and The Belfast Link

Laboratories, Belfast, Northern Ireland, UK

D T McManus

A H Patterson

P Maxwell

M W Humphreys

N H Anderson

Correspondence to:

Dr D T McManus,

Department of Pathology,

Queen's University of

Belfast, Royal Group of

Hospitals Trust, Belfast

BT12 6BA, Northern

Ireland, UK.

email:dmcmanus@qub.ac.uk

Accepted for publication 29 October 1998 oncogene occurs in $\sim 21 \%$ of cancers, and that such overexpression is associated with more biologically aggressive tumours. Amplification and/or overexpression is also seen in ductal carcinoma in situ, particularly comedo and other high grade subtypes. ${ }^{6}$ Controversy surrounds the value of erbB2 as a prognostic marker in relation to responses to chemotherapy and it has been suggested that the investigation of patients receiving neoadjuvant chemotherapy might help to resolve this issue. ${ }^{5}$ In this context, preoperative detection of erbB2 amplification in breast cancer using fine needle aspirates ${ }^{78}$ might be of particular relevance and could be useful clinically. Aspirates contain whole unfixed tumour cell nuclei and in many ways are ideal specimens for fluoresence in situ hybridisation (FISH) analysis.

A rapid and simple qualitative method for detecting amplification of the erbB2 gene in breast cancer fine needle aspirates is described and the results are correlated with conventional immunohistochemical staining of paraffin wax embedded sections with antibody CB11. Hybridisation of a chromosome 17 centromere probe allowed comparisons to be made between erbB2 copy number and chromosome 17 centromere number.

\section{Materials and methods}

To confirm the specificity of the chromosome 17 centromere and erbB2 probes they were co-hybridised to metaphase spreads prepared from a normal individual by conventional methods.

Fine needle aspirates were prepared from 15 unfixed operative breast cancer specimens. The aspirates were smeared on to a small area of APES coated slides. The slides were denatured in $70 \%$ formamide, $2 \times$ saline sodium citrate (SSC) at $70^{\circ} \mathrm{C}$, plunged into $70 \%$ ice cold ethanol, transferred to ethanol at room temperature, and allowed to dry. A digoxigenin labelled probe to the erbB2 locus (Oncor, Durham, UK) was prewarmed to $37^{\circ} \mathrm{C}$ and hybridised overnight at $37^{\circ} \mathrm{C}$ in a moist chamber. The stringency washes in $50 \%$ formamide, $1 \times \mathrm{SSC}$, at $37^{\circ} \mathrm{C}$, were followed by a one step indirect detection method using antidigoxigenin fluorescein isothiocyanate (FITC) or antidigoxigenin rhodamine for the single and dual hybridisation experiments, respectively.

For the dual hybridisation experiments, a centromere probe directly labelled with FITC (Boehringer Mannheim, Lewes, Sussex, UK) was used. The slide and the centromere probes were denatured separately; the centromere and the erbB2 probes were then applied sequentially. The erbB2 probe was detected using antidigoxigenin rhodamine (at a dilution of $1 / 200)$. 


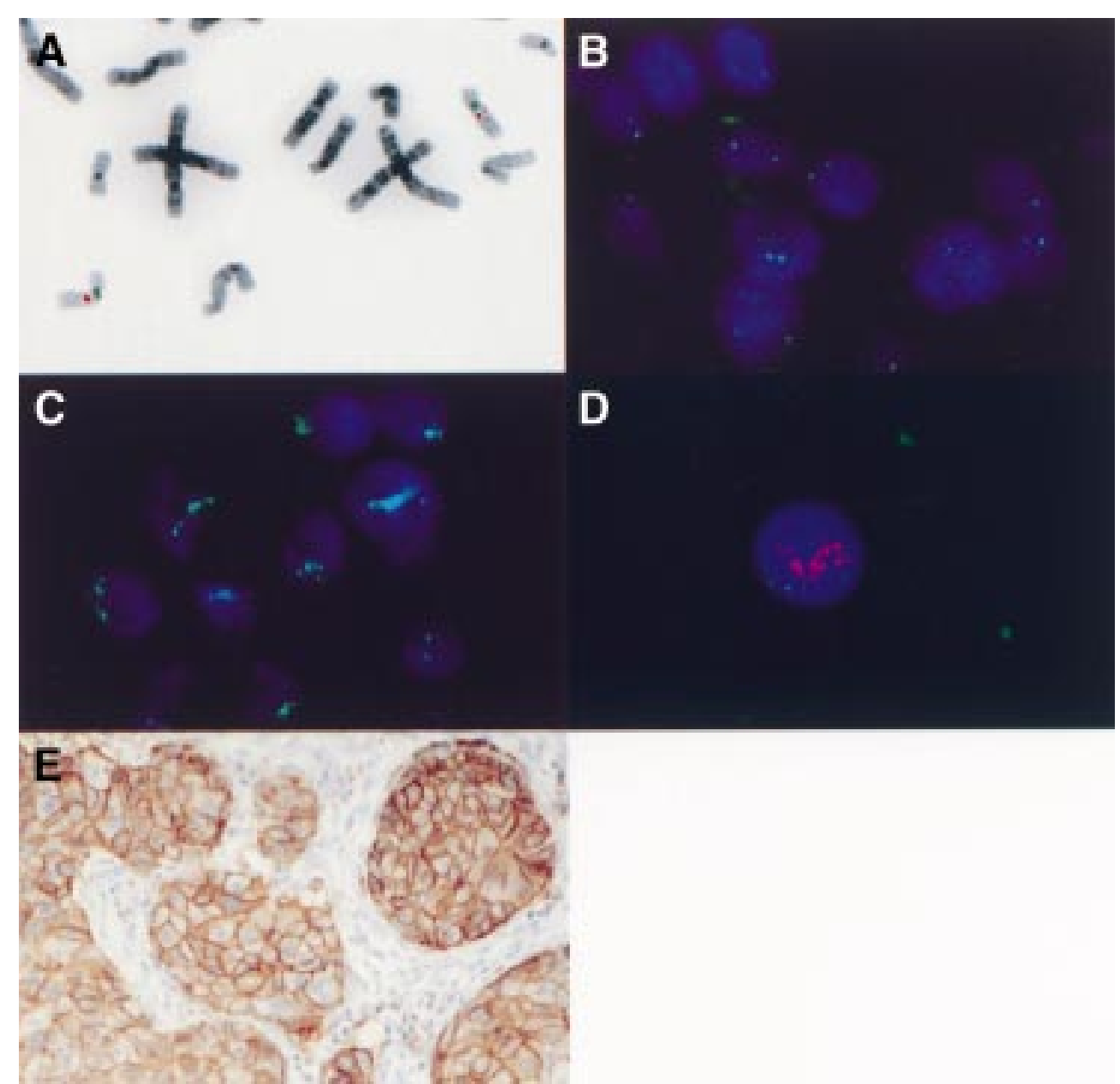

Figure 1 (A) Dual hybridisation to a metaphase spread: chromosome 17 centromere probe, green signal; erbB2 probe, red signal. (B) erbB2 probe (green signal) hybridised to interphase breast cancer cell nuclei: no amplification detected. (C) erbB2 probe (green signal) hybridised to interphase breast cancer cell nuclei: amplification detected. (D) Dual hybridisation to interphase breast cancer cell nucleus: erbB2 amplified (red signals), chromosome 17 centromeric probe (green signal). (E) Diffuse membrane staining with antibody CB11 in a paraffin wax embedded section of an infiltrating ductal carcinoma.

In seven cases, the erbB2 probe was detected by antidigoxigenin FITC and a digoxigenin labelled centromere probe (Boehringer Mannheim) was hybridised separately to a different slide from the same case.

The counterstain used in all specimens was DAPI (4,5 diamino-2-phenyl-indole. The slides were examined at $\times 1000$ magnification using a Leica epifluorescence microscope with a triple band pass filter set. Fluorescence signals were collected through a Photometrics (Tucson, Arizona, USA) black and white cooled charged coupled device (CCD) camera linked to an Apple Mac (Apple Computer Company, Middlesex, UK) computer running the Vysis smart capture system, enabling the capture of digitised colour fluorescence images. The FITC, rhodamine, and DAPI signals were viewed and captured using the respective excitation filters. All three channels were captured consecutively and pseudocoloured to their respective primary colours (rhodamine, red; FITC, green; DAPI, blue) and automatically merged using the smart capture software.

Paraffin wax embedded sections of the corresponding cancers were stained with monoclonal antibody CB11 (NovoCastra, Newcastle upon Tyne, UK) at a 1/50 dilution after microwave antigen retrieval, using $0.1 \mathrm{M}$ citrate buffer, $\mathrm{pH}$ 6.0, for 20 minutes. A standard streptavidin biotin complex method (Dako, Ely, Cambridgeshire, UK) was used with peroxidase/diaminobenzidine as chromogen.

\section{Results}

Dual hybridisation of the chromosome 17 centromere/erbB2 probe to metaphase spreads from a normal individual (fig 1A) confirmed the specificity of both probes under the stringency conditions described.

As expected, the signal intensity of the erbB2 probe was less than that of the chromosome 17 centromere probe, so that fewer cells showed two clearly visible signals in the aspirates not showing amplification (fig 1B).

Three of the 15 aspirates showed high level erbB2 amplification as detected by FISH. In the amplified cases, the amplified erbB2 signals were evident as clusters of intranuclear pink or green dots, depending on the detection system (fig 1C and D), and in keeping with amplification by a mechanism involving homogenously staining regions. ${ }^{7}$ These three specimens were also polysomic for chromosome 17 . It was possible to discern extra copies of erbB2 in other aspirates polysomic for chromosome $17 \mathrm{but}$, on 
inspection, it was easy to distinguish these from the specimens showing high level amplification.

Three of the 15 tumours showed diffuse staining of the tumour cell membranes with antibody CB11 (fig 1E). The other 12 tumours showed no evidence of immunoreactivity. The same three tumours also showed evidence of erbB2 amplification by FISH in the aspirates.

\section{Discussion}

Amplification of the erbB2 gene can be detected by solid matrix blotting techniques, such as dot blots and Southern blotting, ${ }^{5}$ or the differential polymerase chain reaction, ${ }^{9}$ using constitutively expressed genes such as actin as an internal control. However, these techniques may underestimate copy number, because the final signal gives an average estimation of the level of amplification in the sample. Amplified signals from the tumour cells might be diluted considerably by contaminating stromal cells that have a normal copy number. Therefore, such approaches are more likely to produce a continuous distribution of amplification levels, with associated difficulties in selecting a threshold for a positive result. FISH is emerging as the most sensitive and reliable technique for detecting amplification of the erbB2 oncogene, at least in paraffin wax embedded material. ${ }^{4}$ Moreover, FISH may also allow studies into tumour heterogeneity with respect to gene amplification.

In our investigation, amplified cases contained multiple copies of the erbB2 locus, clustered together, in keeping with amplification in the form of intrachromosomal homogenously staining regions, rather than double minutes. ${ }^{7}$ Such cases were easily separated by inspection from aneuploid cases containing several copies only of the erbB2 locus. Comparison with the 17 centromere copy number was helpful in this respect but not essential. Only the three cases with multiple copies of the gene showed diffuse membrane staining with antibody CB11. A quantitative estimation of an erbB2 to chromosome 17 centromere copy number ratio, as in previous investigations was not necessary. ${ }^{78}$ Visualisation of the erbB2 signals in unamplified specimens was difficult in some instances, particularly when examining the slides using a conventional epifluorescence microscope without the benefit of digital image capture, enhancement, and storage afforded by the smart capture system. The use of amplification methods such as biotinylated tyramide may facilitate the visualisation of such weak cosmid signals.
The FISH assay is completed in two days and is not much more labour intensive than immunohistochemical techniques. The procedure could be completed within a single working day if necessary. The commercial probe is very expensive, but the costs could be reduced greatly if probes were purified and labelled in house. The specialised fluorescence imaging systems are very expensive and time consuming to use.

In conclusion, our pilot study has shown that amplification of the erbB2 oncogene can be detected by FISH using fine needle aspirates from resected breast cancers. The technique should be readily applicable to clinical fine needle aspirates. Previously, we have detected numerical chromosomal abnormalities in fine needle aspirates from palpable tumours and radiologically guided aspirates obtained from the screening programme. ${ }^{10}$ Amplification of the gene correlates with protein overproduction, detected by immunohistochemistry as diffuse membrane staining in paraffin wax embedded sections. Amplification is readily detectable qualitatively by inspection without dual hybridisation and calculation of an erbB2 to chromosome 17 centromere copy number ratio.

This work was supported by grants from Action Cancer and Cansearch to DT McManus and NH Anderson, respectively.

1 Poescu NC, King CR, Kraus MH. Localisation of the human erbB-2 gene on normal and rearranged chromosomes 17 to bands q12-21.32. Genomics 1989;4:362-6.

2 Cousens L, Yang-Feng TL, Chen YC, et al. Tyrosine kinase receptor with extensive homology to EGF receptor shares chromosomal location with neu oncogene. Science 1985; 230:1132-9.

3 Slamon DJ, Clark GM, Wong SG, et al. Human breast cancer: correlation of relapse and survival with amplification of the HER-2/neu oncogene. Science 1987;235:17782 .

4 Pauletti G, Godoplhin W, Press MF. Detection and quantitation of HER-2/neu gene amplification in human breast cancer archival material using fluorescence in situ hybridisation. Oncogene 1996;13:63-72.

5 Revillion F, Bonneterre J, Peyrat JP. ERBB2 oncogene in human breast cancer and its clinical significance. European fournal of Oncology 1998;34:791-808.

6 Sommerville JE, Clarke LA, Biggart JD. c-erbB2 overexpression and histological types of invasive breast carcipression and histological types of

7 Kallioniemi O, Kallioniemi A, Kurisu W, et al. C-erbB-2 oncogene amplification in breast cancer analysed by luorescence in situ hybridisation. Proc Natl Acad Sci USA 1992;89:5321-5.

8 Sauter G, Feichter G, Torhorst MD, et al. Fluorescence in situ hybridisation for detecting erb2 amplification in breast tumour fine needle aspiration biopsies. Acta Cytologica 1996;40:164-73.

9 An HX, Niederacher D, Beckmann MW, et al. ERBB2 gene amplification detected by fluorescent differential polymerase chain reaction in paraffin-embedded breast carcinoma tissues. Int $\mathcal{F}$ Cancer 1995;64:291-7

10 McManus DT, Patterson AHP, Maxwell P, et al. Interphase cytogenetics of chromosomes 11 and 17 in fine needle aspirates of breast cancer. Hum Pathol. [In press.] 